\title{
Nanodrugs: A dream, the future, or just the coming reality?
}

\section{Diego Ettore Liberati* \\ Dipartimento di Elettronica, Research Director National Research Council of Italy, Milano, Italy}

When thinking to the perfect drug for better therapy one would like to have a molecule able to be speedily administered just to the very target, without collateral effects nor transit compartments to travel through. Traditionally drugs are instead mostly assumed by ingestion, going to blood through digestion, when not directly injected, and then traveling through the entire body implying quite a lot of collateral effects besides therapy. Nanoparticles could be directly injected and produced in such a way to be minimally toxic while active. It is not easy, as proved by several lack of results in the last few years. But sometimes it could be easier, as shown by a great start up company spinning off from University of Padua, thanks to a young bright lady professor: having discovered a molecule against cirrhosis, the problem was how to administer it: embedding it in sugar and injecting in blood, it travels to liver, eager of both blood and sugar thus locally dissolving the sugar cap and exposing the molecule, healing the cirrhotic liver. Columbus' egg: not a general solution, but a general suggestion: make thinks as simple as one could to hope it works. Other cases may be more difficult, requiring a more complex chemical cap and/or more complex physical transport, but the road is traced, toward a true personalized medicine, considering the very need of each patient and avoiding damaging while trying to heal, as per the ancient Hippocratic Oath!
Copyright: $(02018$ Liberati DE. This is an open-access article distributed under the terms of the Creative Commons Attribution License, which permits unrestricted use, distribution, and reproduction in any medium, provided the original author and source are credited.
${ }^{\star}$ Correspondence to: Diego Ettore Liberati, PhD, Research Director National Research Council of Italy, Information \& Control 4 Systems Biology @ IEIIT, Dipartimento di Elettronica, Informazione e Bioingegneria, Politecnico Piazza Leonardo da Vinci 32, Milano, Italy, E-mail: diego.liberati@polimi.it

Received: August 16, 2018; Accepted: August 27, 2018; Published: August 31, 2018 